\title{
El aprendizaje de vocabulario en español como L2 a través del uso de tecnología. Un análisis de género
}

\author{
Silvia Peart \\ United States Naval Academy \\ Argentina
}

Recibido: 20 de junio de $2011 /$ Aceptado: 18 de enero de 2011

ISSN: 1697-7467

\begin{abstract}
RESUMEN: El objetivo de este artículo es determinar si existen diferencias de género en cuanto al proceso de aprendizaje de vocabulario en una L2 que alumnos y alumnas realizaron en una plataforma Web. Para ello, a través de métodos cualitativos y cuantitativos, se analizó el proceso de aprendizaje de un grupo de estudiantes que aprenden español como segunda lengua a nivel universitario en Tejas $(n=18)$. Los resultados de este estudio indican que los ejercicios diseñados en la plataforma Web favorecerían las estrategias de aprendizaje de vocabulario de la población masculina, pero no así las estrategias de la población femenina.

Palabras clave: aprendizaje de vocabulario en español como L2, coeducación, género, aprendizaje de vocabulario en una plataforma Web
\end{abstract}

Learning vocabulary in Spanish as an L2 using technology. A gender analysis.

\begin{abstract}
The main objective of this study is to determine whether there are gender differences regarding the L2 vocabulary learning process which students completed on a Web-based platform. This investigation was conducted with a group of students leaning Spanish as an L2 at university level in Texas $(n=18)$. Qualitative and quantitative analyses indicate that the Web-based activities for vocabulary learning would favor the male population having a «gender effect».

Keywords: learning vocabulary in Spanish as an L2, coeducation, gender, learning vocabulary on a Web-based platform
\end{abstract}

\section{INTRODUCCIÓN}

En los últimos 30 ó 35 años, el campo de adquisición de segunda lengua ha recibido el impacto del ALAO (Aprendizaje de Lengua Asistido por Ordenador). Al final de los 90 el uso del correo electrónico, Internet y tecnología multimedia se ha incrementado dentro de la clase y fuera de ella complementándola (Dodigovic, 2005).

La enseñanza de vocabulario que una vez se realizara en clase, se ha visto enriquecida por el uso de Internet, e-mail, chats, blog, Wiki y herramientas pedagógicas diseña- 
das en diversas plataformas electrónicas que se han convertido en un complemento indispensable del tradicional libro de texto. En esta misma línea, se han desarrollado plataformas educativas como WebCT, Blackboard o Angel, entre otras. Estas herramientas a la vez que, complementan la enseñanza en el aula, se enfocan en la producción escrita u oral o en ambas a la vez, o la sustituyen, como es el caso de las clases a distancia. A lo largo de este artículo analizamos distintos estudios que exploran la utilidad de programas diseñados en ordenadores o ejercicios en plataformas educativas para la adquisición de vocabulario en una segunda lengua.

Estas herramientas pedagógicas han encontrado su fundamento en teorías que destacan la importancia del interaccionismo, ya que Long (1996), por ejemplo, afirma que es en la interacción donde se adquiere una segunda lengua y donde los aprendices «notan» los errores y se negocian nuevas estrategias de adquisición. Se puede decir que ALAO ha optimizado el proceso de adquisición de una segunda lengua (L2), si entendemos que el mismo como un proceso colaborativo (Swain, 2000). ALAO interviene positivamente, incentivando tanto la producción escrita como oral. En este marco, las investigaciones realizadas sobre ALAO (Chang \& Smith, 1991; Chapelle, 1994, 1997, 1998, 2001; Dunkel, 1991; Levy, 1997; Dodigovic, 2005), muestran que la interacción con distintas herramientas multimedia han ayudado en diversas áreas del aprendizaje de una segunda lengua y en particular en la adquisición del vocabulario nuevo. Por ejemplo, Chun (1994) y Kitade (2000) muestran en sus investigaciones los resultados positivos alcanzados en relación a la lecto-comprensión; Pelletieri (2000) los presenta también en relación al desarrollo morfosintáctico; Chun (1994) enfatiza los beneficios conseguidos en la competencia sociolingüística; y Kitade (2000) y Dodigovic (2005), en la calidad y cantidad de producción de output por parte de los aprendices. Todos estos estudios enfatizan aspectos positivos de distintas plataformas desarrolladas en ALAO como materiales de apoyo para la clase en el proceso de adquisición de una segunda lengua. En particular se enfatiza la interacción y la producción oral y escrita que puede favorecer la «atención» a ciertas formas en relación al significado; esto permite que el aprendiz modifique su sistema lingüístico en desarrollo y aprenda de sus propios errores. Las distintas tareas que se proponen a los aprendices en el formato de ALAO les permiten centrarse en aspectos morfológicos y sintácticos, aspectos de la lengua que, de otro modo, pasarían, quizá, inadvertidos. A su vez, estos sistemas se adaptan a las propias necesidades del usuario, al tiempo que este necesita practicar, producir, repensar, re-leer, etc... De este modo, y a través de formatos basados en ALAO, los estudiantes pueden dirigir su propio aprendizaje, auto-corregirse facilitando así el desarrollo de su sistema lingüístico. Este proceso se continúa y profundiza en el aula con la asistencia del profesor, quien puede diseñar ejercicios que se ajusten a las necesidades de sus estudiantes.

Más concretamente en la adquisición del vocabulario, por ejemplo, De la Fuente (2003), desde la perspectiva interaccionista, examina los potenciales efectos de la negociación de significado en el desarrollo de vocabulario en español como segunda lengua y los efectos de CMC (Computer-Mediated Communication) en el proceso de adquisición de vocabulario en español. Por otro lado, Sun, y Dong, (2004) en su estudio construyeron un software para desarrollar el vocabulario en inglés como segunda lengua de niños cuya primera lengua es el chino. En este caso, los autores enfatizan la importancia de proveer a los aprendices no sólo de contextos apropiados, sino también de imágenes, que 
colaboren en el aprendizaje del vocabulario de una segunda lengua. La información que se presenta en videos, en línea, en gráficos, dibujos animados, entre otros, puede crear un contexto multisensorial que colabore en la adquisición del vocabulario de la L2, en especial cuando los aprendices no tienen suficiente input de la lengua objeto en su vida diaria. Sun y Dong (2004), también enfatizan que los medios audiovisuales e incluso los dibujos permiten asociaciones que hacen que los aprendices relacionen formas gramaticales con gráficos, dibujos, música, etc..., lo que les permite adquirir de un modo más significativo el vocabulario nuevo. Tozcu y Coady (2004) enfatizan la importancia del aprendizaje de vocabulario para la comprensión lectora. Ambos desarrollan su investigación en la línea de la adquisición del inglés como segunda lengua y en el desarrollo de programas con ordenadores que mejoren la adquisición de vocabulario en el marco de la comprensión lectora. Los autores afirman que el vocabulario de uso más frecuente es el que primero se adquiere y es justamente el que ayudará a los aprendices a comprender luego un texto escrito, por tanto Tozcu y Coady (2004) proponen presentar de manera interactiva a través de ALAO este vocabulario de uso más frecuente para facilitar su adquisición.

En todos los casos antes presentados los investigadores analizan y desarrollan distintos tipos de sistemas con ordenadores que faciliten el aprendizaje del vocabulario en una segunda lengua fundamentalmente teniendo en cuenta la interacción que el aprendiz ha de realizar con el sistema y posteriormente en el aula con sus compañeros y su profesor. En todos los casos se enfatiza cómo estos sistemas pueden ayudar en la producción de output tanto escrito como oral y la frecuencia de input que pueden ofrecer.

\subsection{Aprendizaje de vocabulario como L2 y género}

El rol crucial que representa el aprendizaje de vocabulario en el marco de la adquisición de segundas lenguas ha sido largamente estudiado tanto en su aspecto teórico como en el práctico (ver por ejemplo, Pavlenko, 1999; Singleton, 1999). A pesar de la importancia que se le otorga a la competencia léxica y al aprendizaje de vocabulario con L2, son escasos los estudios que incorporan el género como una variable a investigar. Dentro de los pocos estudios en este ámbito, encontramos a Jiménez Catalán (2003), quien en su investigación empírica concluye que si bien la población masculina y la femenina usan estrategias semejantes para la adquisición del vocabulario, la población femenina usa un abanico mayor de estrategias y más variadas que la población masculina. En su estudio, Jiménez Catalán (2003) sostiene que la población femenina emplea estrategias sociales que promueven la comunicación y la interacción, la provocación y el monitoreo; mientras que la población masculina usa estrategias de traducción, y relaciona el vocabulario con representaciones del mismo. Es importante señalar que los resultados del estudio de Jiménez Catalán (2003) muestran un acercamiento diferente al aprendizaje de vocabulario de la L2 por parte de la población femenina y la masculina. En un estudio posterior, Jiménez Catalán (2005) también muestra la ausencia de bases de datos que relacionen el género y la adquisición de segundas lenguas. Concretamente, ella afirma que esta ausencia constituye un círculo vicioso que a su vez dificulta las investigaciones en esta área.

Es interesante destacar que, en general, las investigaciones sobre la adquisición de vocabulario como L2 no incluyen al género como una variable ni tampoco diferencian el 
tipo de estrategias que la población masculina y la femenina emplean en el proceso de aprendizaje del mismo, es por esta razón por la que el presente estudio incluirá esta variable y se tendrá en cuenta como eje a la hora de analizar los datos recogidos.

\subsection{Motivación del estudio}

Explorar y evaluar la posible correlación de los ejercicios diseñados en la plataforma educativa WebCT, el tiempo que emplean en ellos los estudiantes y su desempeño posterior en un examen parcial. Analizar y evaluar si existe un «efecto de género» en el trabajo que las alumnas y los alumnos desempeñan en la plataforma educativa WebCT y su rendimiento académico en los exámenes parciales.

\subsection{Preguntas del Estudio}

1. ¿Los estudiantes del curso Español Intermedio I que pasan más tiempo practicando el vocabulario en la plataforma educativa WebCT obtienen mejores resultados que los que practican menos tiempo?

2. ¿La cantidad de veces que los estudiantes hacen los ejercicios en la plataforma educativa WebCT los ayuda a aprender mejor el vocabulario? ¿Esto se refleja en los exámenes parciales?

3. ¿Existe una correlación positiva o negativa entre las notas que los estudiantes obtienen en los ejercicios en la plataforma educativa WebCT, las notas de los exámenes y el tiempo que trabajan en línea? ¿Se corresponden estos resultados con sus propias percepciones de los ejercicios en la plataforma educativa WebCT?

4. ¿Existe un «efecto de género» en el rendimiento académico de los estudiantes tanto en la plataforma educativa WebCT como en los exámenes parciales?

\section{Materiales Y MÉtodo}

\subsection{Participantes}

Los participantes de este estudio son 18 estudiantes que se encuentran cursando Español Intermedio I (SPAN 2301) en una Universidad de Tejas, Estados Unidos. En esta universidad, la Facultad de de Artes y Ciencias requiere que todos sus alumnos y alumnas estudien una lengua extranjera como requisito para su graduación, por tanto el Departamento de Lenguas Extranjeras ofrece una serie de cursos que los estudiantes escogen para cumplimentar este requisito dentro de su carrera. Por ser Tejas un estado cercano a la frontera con México, la mayor parte de la población estudiantil escoge cursar español como lengua extranjera. Es por esta razón por la que la División de Español ofrece tres semestres de lengua, con lo cual los estudiantes de la Facultad de de Artes y Ciencias cumplimentan su currículum. El primer semestre de lengua correspondiente al «primer año de español» es lo que en esta institución recibe el nombre de SPAN 1507. Para cursar esta clase los estudiantes deben haber tomado antes por lo menos dos años de español en la escuela secundaria. Luego, lo que se conocerá como «segundo año de 
español» se divide en dos semestres: un curso es Español Intermedio I (SPAN 2301) y Español Intermedio II (SPAN 2302). SPAN 1507 es un curso de 5 créditos-horas mientras que SPAN 2301 y SPAN 2302 son cursos de 3 créditos-horas respectivamente.

Durante los últimos años estos cursos han sido rediseñados paulatinamente y transformados en clases híbridas, contando con horas presenciales y horas que los estudiantes pasan trabajando en la Web usando la plataforma educativa WebCT. En el caso del primer curso SPAN 1507, los estudiantes se reúnen con su profesor tres horas por semana y deben pasar dos horas semanales completando ejercicios en WebCT. Para la clase Español Intermedio I y II, los estudiantes deben reunirse con el docente dos horas por semana y pasar una hora semanal trabajando en los ejercicios diseñados en WebCT. En ambos casos, estos ejercicios están íntimamente relacionados con el libro de texto que se usa en la clase; algunas actividades enfatizan el uso vocabulario de de ese capítulo y otras los aspectos gramaticales que se cubrirán en una determinada lección.

El presente estudio se enfocará en el curso Español Intermedio I (SPAN 2301), en una sección en particular. Para realizar este estudio se contó con aprobación de la oficina del Programa de Protección de la Investigación de personas (Human Research Protection Program. (HRPP Office) de esta institución. El grupo de estudiantes fue seleccionado al azar en conjunción con el coordinador del nivel y el profesor del curso.

El grupo de participantes fue observado a lo largo de dos semanas mientras se desarrolló el capítulo 9 del libro Puntos de Partida $7^{1}$. Se recabaron datos de los participantes a través de una encuesta realizada al terminar este capítulo.

Como parte de la investigación también se incluyó al docente de esta sección, quien fuera entrevistado en repetidas ocasiones, primero en relación al material utilizado en clase y en relación a sus propias convicciones sobre la enseñanza del vocabulario en clase y en la plataforma educativa WebCT; y luego en relación al rendimiento académico de sus estudiantes tanto en el trabajo en la plataforma Web como en el primer examen parcial. El profesor de esta sección es un hablante nativo del español, estudiante de posgrado del programa de español y hace dos años que enseña en esta institución.

\subsection{Técnica de Recogida de Datos}

Para este estudio se desarrollaron encuestas estructuradas y semi-estructuradas. La primera se aplicó a los estudiantes, con el fin de que pudieran contestarla en poco tiempo siendo lo más concisos posible. El segundo tipo de encuesta se aplicó al profesor.

La primera entrevista semi-estructurada tenía como objetivo recavar información acerca del docente, su filosofía de enseñanza en relación con el vocabulario, su visión respecto a los ejercicios en la plataforma educativa WebCT y conocer cómo este establece o no relaciones entre estos ejercicios y el vocabulario que se enseña en clase y cómo aprenden los estudiantes el vocabulario nuevo de la lección.

${ }^{1}$ En este capítulo el vocabulario que se presenta tiene relación con las fiestas y festejos en el mundo hispano. También se introduce el pretérito imperfecto como punto gramatical fundamental. 


\subsection{Procedimientos}

Para llevar a cabo este estudio se realizaron observaciones de clase durante dos semanas. Los estudiantes de este curso se reunían dos veces por semana: martes y jueves de 10.00 a 10.50 cada día. En estas observaciones se registraron todas las interacciones realizadas entre los estudiantes y el profesor, las actividades realizadas y el número de estudiantes presentes en cada clase cada día de la observación. Inmediatamente después de cada clase, se enviaban las observaciones por correo electrónico tanto al profesor como a dos estudiantes seleccionados al azar para obtener feedback en relación al registro y validarlo a través de tres observadores participantes en el estudio. Este procedimiento se repitió durante las dos semanas que en que llevó a cabo este proyecto. Durante este tiempo, las clases se impartieron en el aula regularmente y no hubo exámenes.

Al finalizar el capítulo 9, cuyo eje era el vocabulario de «días festivos», se realizó una encuesta a los estudiantes. Antes de que la contestaran se les explicó que esta encuesta era parte de un estudio que buscaba mejorar la enseñanza del vocabulario en Español Intermedio I (SPAN 2301), que sería totalmente confidencial, que su profesor no tendría acceso a los resultados de la misma y que dicha encuesta no alteraría las notas que recibirían en el curso. Las mismas se repartieron y se pidió al docente que abandonara el aula mientras los participantes respondían. Al finalizarla y, una vez recogidas las mismas, el profesor volvió a la clase.

Dos semanas más tarde, se recogieron los resultados de los ejercicios en la plataforma educativa WebCT del capítulo 9 realizados por los estudiantes. Estos ejercicios son parte de la tarea regular que ellos necesitan realizar para obtener crédito a lo largo del curso. Los mismos están diseñados en WebCT y sus resultados quedan grabados en esta plataforma. De allí se exportaron los datos a una planilla de cálculo, se asignó un número a cada estudiante para garantizar su anonimato, se registró cuántas veces realizó los ejercicios en la Web, cuál fue su nota cada vez que los realizó, cuál fue su nota final y cuánto tiempo pasó cada estudiante trabajando activamente en línea. Una vez realizado esto, se le solicitó al profesor los resultados de los exámenes parciales, que también se colocaron en una planilla de cálculos, discriminando la nota que obtuvieron los estudiantes en el apartado de opción múltiple del examen y en la composición.

Una vez que se obtuvo toda la información referente a los ejercicios en la plataforma educativa WebCT, a los exámenes y a las observaciones en clase, se realizaron diferentes entrevistas con el profesor. La primera para obtener información referente a su propio posicionamiento en relación a los ejercicios en la plataforma educativa WebCT y la enseñanza del vocabulario y luego se realizaron otras entrevistas más orientadas a recabar información acerca de los estudiantes y su desempeño en los ejercicios en la plataforma educativa WebCT, en clase y los exámenes. Para ello, se analizó la nota de cada ejercicio con el docente así como las notas de la composición y el primer examen parcial del curso. Cada entrevista con el docente fue grabada en su totalidad. 


\section{Resultados}

Para realizar el análisis de los datos recabados se dividió a la población masculina de la femenina. Siguiendo esta línea de investigación decidimos tabular los resultados de cada población por separado y luego compararlos en cada caso para verificar si existían diferencias en relación a los datos obtenidos.

De las observaciones de clase y de las repetidas entrevistas realizadas con el docente se puede decir que la población femenina ( 9 estudiantes de un total de 18) participa más activamente en la clase en interacción con el profesor. La población femenina, responde a sus preguntas en español sin necesidad de que el docente las llame por su nombre o les haga preguntas directamente. En general durante las dos semanas de observación, la población femenina se mostró cordial con su profesor, se ofrecía a participar voluntariamente y se pudo contar con un mayor número de interacciones entre la población femenina que entre la población masculina. La población masculina, por el contrario, sólo participaba en la clase si el profesor les hacía una pregunta directa o los llamaba por su nombre. También la población masculina se mostró cordial con su profesor pero más reticente a hablar en español en la clase. Sin embargo, a pesar de estos resultados preliminares, al analizar los datos de los ejercicios en la plataforma educativa WebCT y del primer examen parcial se pudo observar que la población masculina obtuvo mejores notas que la población femenina en todos los casos; así nos lo muestra la tabla 1 .

Tabla 1. Media de las notas en los ejercicios de WebCT y el examen. Población masculina y femenina

\begin{tabular}{|l|c|c|c|c|c|c|}
\hline & $\begin{array}{c}\text { Ejercicios } \\
\text { en WebCT } \\
1\end{array}$ & $\begin{array}{c}\text { Ejercicios } \\
\text { en WebCT } \\
2\end{array}$ & $\begin{array}{c}\text { Lectura } \\
\text { en } \\
\text { WebCT }\end{array}$ & $\begin{array}{c}\text { Horas } \\
\text { trabajadas } \\
\text { en } \\
\text { WebCT }\end{array}$ & $\begin{array}{c}\text { Examen } \\
\text { parcial 1 }\end{array}$ & $\begin{array}{c}\text { Composición } \\
\text { del Examen } \\
\text { parcial 1 }\end{array}$ \\
\hline ALUMNAS(9) & $\mathbf{5 9 . 2}$ & $\mathbf{8 8 . 2}$ & $\mathbf{8 8}$ & $\mathbf{9}$ & $\mathbf{6 9 . 1}$ & $\mathbf{9 2}$ \\
\hline ALUMNOS(9) & $\mathbf{9 3 . 6}$ & $\mathbf{8 9 . 4}$ & $\mathbf{9 0}$ & $\mathbf{1 1}$ & $\mathbf{7 0 . 9}$ & $\mathbf{9 4}$ \\
\hline
\end{tabular}

Conforme se ve en la misma, la población masculina obtuvo un mejor rendimiento académico que la población femenina en todas las áreas. Tres alumnas no realizaron los ejercicios en la plataforma educativa WebCT de esa semana y obtuvieron ceros, de ahí el contraste entre la media de alumnos y alumnas. En los otros ejercicios, lectura y examen no hay diferencias tan profundas pero aun así la población masculina obtiene resultados favorables y también muestra mayor tiempo de trabajo en la plataforma Web: la población masculina trabajó un total de 11 horas en WebCT en dos semanas y la población femenina trabajó 9, dos horas menos. De modo que si bien la población femenina interacciona más en clase y, según dijera el profesor del curso, «participaban más,» es la población masculina la que pasa más tiempo fuera de clase trabajando en la plataforma Web y obtiene mejores notas en este tipo de ejercicios que la población femenina. Debido a los resultados preliminares que obtuvimos, se decidió analizar las notas del primer examen oral, cuyos resultados se representan en la tabla 2 . 
Tabla 2. Media y desviación estándar del total de notas en el examen oral

\begin{tabular}{|l|c|c|}
\hline & ALUMNAS & ALUMNOS \\
\hline Examen oral. Media & $\mathbf{7 6}$ & $\mathbf{7 4}$ \\
\hline $\begin{array}{l}\text { Examen oral. } \\
\text { Desviación Estándar }\end{array}$ & $\mathbf{8 . 4 3}$ & $\mathbf{1 3 . 5 0}$ \\
\hline
\end{tabular}

Tal y como refleja la tabla 2, la población femenina obtiene mejores resultados en el primer examen oral que la población masculina (76 vs.74), la media en el caso de las alumnas es mayor y la desviación estándar (8.43) muestra una dispersión menor en torno a la media entre la población femenina. Esto se corresponde con las opiniones del profesor y con las observaciones realizadas en clase donde registramos mayores interacciones entre la población femenina que entre la masculina. En relación a la población masculina se obtienen mejores resultados en los ejercicios en línea que tienen que ver con la producción escrita y no con la producción oral. Estos resultados parecen sugerir que la población femenina «prefiere» la interacción en clase a la hora de aprender vocabulario nuevo. Las alumnas interaccionan en la clase entre ellas y con su profesor para aprender el vocabulario de la lección mientras que los alumnos se muestran más «pasivos», aunque luego vemos que ellos optan por actividades más solitarias que tienen que ver con la traducción o la identificación de imágenes con el vocabulario adecuado (matching activities). Esta manera diferente de interaccionar parecería corroborar las hipótesis de Jiménez Catalán (2003) en relación a las diferentes estrategias para el aprendizaje de vocabulario. Al parecer, el diseño de las actividades en la plataforma educativa WebCT favorecería estrategias masculinas de aprendizaje de vocabulario como L2, probablemente es por esta razón por la que los alumnos pasan más tiempo trabajando en la plataforma educativa WebCT.

Para ahondar en las percepciones de los alumnos y alumnas en relación a las actividades en WebCT y en general a la tecnología usada en este curso, se recogieron y analizaron las encuestas realizadas a los estudiantes. Los datos más significativos se presentan en la tabla 3.

Tabla 3. Selección de preguntas de la encuesta realizada a los studiantes del curso

\begin{tabular}{|l|l|c|c|c|}
\hline $\begin{array}{l}\text { Preguntas de la encuesta } \\
\text { realizada a toda la clase }\end{array}$ & \multicolumn{1}{|c|}{ Opciones } & $\begin{array}{c}\text { ALUMNAS } \\
9\end{array}$ & $\begin{array}{c}\text { ALUMNOS } \\
9\end{array}$ & $\begin{array}{c}\text { TOTAL } \\
18\end{array}$ \\
\hline $\begin{array}{l}\text { 5.1 Los ejercicios en línea } \\
\text { me ayudan a aprender el }\end{array}$ & Agree \& strongly agree & $55 \%$ & $\mathbf{8 8 \%}$ & $72 \%$ \\
vocabulario de la lección & Somewhat agree & $22 \%$ & $11 \%$ & $16 \%$ \\
\hline 5.6 En general los ejercicios & Disagree & $22 \%$ & $0 \%$ & $11 \%$ \\
$\begin{array}{l}\text { en línea me ayudan a a strongly agree } \\
\text { aprender español }\end{array}$ & Somewhat agree & $35 \%$ & $\mathbf{8 8 \%}$ & $72 \%$ \\
& Disagree & $11 \%$ & $0 \%$ & $22 \%$ \\
\hline
\end{tabular}

En el extracto de la encuesta realizada, podemos ver que en la pregunta 5.1 un $88 \%$ de la población masculina está de acuerdo en que los ejercicios en WebCT les ayudan a aprender el vocabulario de la lección, mientras que sólo un $55 \%$ de la población femenina está de acuerdo con la misma premisa. Esta afirmación que hace la población 
masculina se corresponde con el hecho de que los alumnos pasan mayor tiempo trabajando en WebCT y luego obtienen mejores notas en estos ejercicios, de modo que los alumnos perciben éstos como facilitadores del aprendizaje de vocabulario. Sólo un poco más de la mitad de la población femenina (55\%) piensa que los ejercicios las ayudan a aprender el vocabulario de la lección, esto también se corresponde con que las alumnas trabajan menos tiempo en línea que los alumnos y obtienen notas más bajas.

Es importante destacar el porcentaje de alumnos y alumnas que no concuerda con esta misma afirmación en cada caso: las alumnas muestran un desacuerdo en un $22 \%$ mientras que entre los alumnos hay un cero por ciento. La población masculina de un modo más moderado acuerda con que los ejercicios en WebCT les ayudan a aprender el vocabulario de la lección, mientras que la población femenina tiene una perspectiva más negativa al respecto.

Otra respuesta interesante de analizar es la de la pregunta 5.6 al final de la tabla 3, donde, nuevamente, un $88 \%$ de la población masculina afirma que los ejercicios en línea en general les ayudan a aprender español, mientras que sólo un 55\% de la población femenina está de acuerdo con esa afirmación. También en este caso, el porcentaje que no coincide con esta afirmación dentro de la población femenina es más alto que entre la población masculina ( $11 \%$ en un caso y cero por ciento en el otro). Es interesante notar que en esta pregunta el porcentaje de alumnas que están en desacuerdo con la afirmación es menor que en la pregunta 5.1. En este caso, la población femenina sigue teniendo una percepción más negativa de los ejercicios en WebCT aunque es más moderada.

A partir de estos resultados, se resolvió analizar una encuesta que el programa de español aplica a todos los estudiantes que cursan Español Intermedio I. En ella se pregunta a los estudiantes sobre la tecnología que se usa en estas clases. En la tabla 4 damos a conocer los resultados más relevantes.

Tabla 4. Resultado de la percepción acerca de la tecnología usada en las clases

\begin{tabular}{|l|l|l|}
\hline & ALUMNAS & ALUMNOS \\
\hline I can learn from an on-line & $\mathbf{5 5 \%}$ agree or strongly agree & $\mathbf{8 8 \%}$ agree or strongly agree \\
course & $\mathbf{3 3 \%}$ do not know & $\mathbf{0 \%}$ do not know \\
& $\mathbf{1 1 \%}$ disagree & $\mathbf{1 1 \%}$ disagree \\
\hline I can learn from a strongly & $\mathbf{8 8 \%}$ agree or strongly agree & $\mathbf{6 0 \%}$ agree or strongly agree \\
computer dependent course & $\mathbf{0 \%}$ do not know & $\mathbf{2 0 \%}$ do not know \\
& $\mathbf{1 1 \%}$ disagree & $\mathbf{2 0 \%}$ disagree \\
\hline
\end{tabular}

En esta tabla encontramos respuestas más que interesantes; la población masculina, en un $88 \%$, afirma que puede aprender de un curso en una plataforma web mientras que sólo un 55\% de la población femenina está de acuerdo con esta afirmación. En general, la población femenina se muestra más reticente y dubitativa para cursar clases en una plataforma web, un $33 \%$ de ella responde que no sabe, mientras que, en el caso de la población masculina, se pronuncian por sí o por no. Las respuestas y los porcentajes en ambos casos se corresponden con las encuestas aplicadas a los estudiantes para este estudio en particular, y también con los resultados de los ejercicios en WebCT y el primer examen parcial. Pero lo más interesante es la segunda afirmación que encontramos en la 
tabla; la población femenina, en un $88 \%$, afirma que pueden aprender de un curso que depende fuertemente de tecnología para su funcionamiento [«strongly computer dependent»] definiéndose esto en la encuesta misma que responden los estudiantes como un curso que requiere el uso de alguna forma de tecnología por lo menos una hora a la semana [«by stronlgy computer dependant course we mean a course that requires use of some form of computer technology at least one hour a week»]. De modo que la población femenina en un porcentaje alto (88\%) afirma que puede aprender de un curso que depende fuertemente de la tecnología, entendiéndose por tal un curso que al menos requiere de algún tipo de ejercicio en computadora al menos una hora por semana, mientras que, de la población masculina, sólo lo afirma un $60 \%$. Es interesante que la población femenina esté en desacuerdo sólo en un $11 \%$ y la población masculina se encuentre más dubitativa y esté en desacuerdo en un $20 \%$. Tal vez si relacionamos las encuestas que se diseñaron en particular para este curso y las que se recogieron sobre la tecnología que se usa en SPAN 2301, podríamos concluir que tanto la población femenina como la masculina no están en desacuerdo en el uso de tecnología en el proceso de aprendizaje del vocabulario en español, pero sí tendrían posiciones divergentes en tanto que la población femenina prefiere una mayor interacción en clase. Basamos esta conclusión preliminar en el hecho de que la población femenina sólo en un 55\% encuentra que los ejercicios en la plataforma educativa WebCT «las ayudan» a aprender español y en que este grupo también en un 55\% afirma que puede aprender de un curso en una plataforma web mientras que un $88 \%$ de la población masculina afirma que los ejercicios en una plataforma en la web los ayudan a aprender español y también en un $88 \%$ que pueden aprender de un curso en la web. Tal vez se puede concluir, basándonos en estas encuestas y en los resultados de los ejercicios en WebCT y el primer examen parcial, que la tecnología no es un problema; quizás la población femenina está requiriendo más interacción para aprender el vocabulario nuevo, una interacción cara a cara y no a través de ordenadores. Pero esto sólo podemos enunciarlo en forma de hipótesis, es necesario desarrollar más estudios empíricos al respecto para alcanzar resultados más concluyentes.

Finalmente presentamos y analizamos las correlaciones estadísticas realizadas entre los ejercicios en la plataforma Web entre sí, con la lectura y el primer examen parcial del curso, seguidamente exponemos estos resultados en la tabla 5.

Tabla 5: Resultados del análisis estadístico Pearson Product Moment para alumnos y alumnas

\begin{tabular}{|l|l|l|}
\hline & ALUMNAS & ALUMNOS \\
\hline Ejercicio en línea 1 y 2 & 0.30 & 0.56 \\
\hline Ejercicio en línea 1 y lectura & 0.20 & 0.47 \\
\hline Ejercicio en línea 1 y examen 1 & $\mathbf{- 0 . 5 5}$ & $\mathbf{0 . 7 2}$ \\
\hline $\begin{array}{l}\text { Ejercicio en línea 1 y la composición } \\
\text { del examen 1 }\end{array}$ & -0.022 & 0.37 \\
\hline Ejercicio en línea 2 y lectura & 0.33 & $\mathbf{0 . 7 9}$ \\
\hline Ejercicio en línea 2 y examen 1 & 0.08 & 0.46 \\
\hline $\begin{array}{l}\text { Ejercicio en línea 2 y la composición } \\
\text { del examen 1 }\end{array}$ & $\mathbf{- 0 . 0 5 8}$ & 0.10 \\
\hline Lectura y examen 1 & -0.032 & $\mathbf{0 . 7 9}$ \\
\hline Lectura y composición & -0.017 & 0.22 \\
\hline Examen 1 y composición & 0.040 & 0.60 \\
\hline
\end{tabular}


En ella, se muestran las correlaciones a partir de la aplicación de Pearson Product Moment en cada resultado de los ejercicios analizados. En el caso de la población masculina, obtenemos una fuerte correlación positiva, como por ejemplo entre el ejercicio en línea 1 y el examen $1^{2}$ donde el resultado es de 0.72 ; el ejercicio en WebCT 2 y la lectura (en línea) la correlación es de 0.79 y la lectura y el examen 1 la correlación es de 0.79 . Esto muestra que hay una fuerte correlación positiva entre los resultados obtenidos por los alumnos en:

- Los ejercicios en línea 1 y la porción del examen 1

- Los resultados de los ejercicios en línea 2, la lectura realizada en WebCT y entre la lectura realizada en WebCT

- La lectura y el examen 1

Esta correlación positiva indica que cuando los resultados de los ejercicios en WebCT 1 son efectivos en términos de notas, también aumentan los resultados del examen 1. La misma lógica se aplicaría en los otros casos. Cuando una variable aumenta (la nota es mayor), la otra también aumenta (la nota también es alta). Esto indicaría que existe una correlación positiva entre el trabajo en WebCT y el examen parcial en el caso de la población masculina.

Para la población femenina tenemos resultados casi opuestos, ya que nótese que las correlaciones obtenidas no son significativas y en caso de serlo son negativas. Por ejemplo, en el caso del ejercicio en WebCT 1 y examen 1 la correlación es de -0.55 y en el caso de los ejercicios en WebCT 2 y la composición del examen 1 la correlación es de -0.058. En ambos casos la correlación no es suficientemente significativa, pero lo curioso es que sea negativa, lo que indica que cuando un valor aumenta el otro disminuye; esto significa que:

- Las alumnas obtuvieron bajos resultados en el ejercicio en WebCT 1, pero mejores resultados y más altos en el examen 1.

- Las alumnas obtuvieron bajos resultados en el ejercicio en WebCT 2, pero mejores resultados y más altos en la composición del examen 1.

Esto, en parte, puede llevar a diversas interpretaciones; por ejemplo, que los resultados de los ejercicios en WebCT no reflejan lo que la población femenina ha estudiado o ha aprendido, en términos de vocabulario. También es ponderable que la población femenina haya desarrollado otras estrategias para el aprendizaje del vocabulario que los ejercicios en WebCT no logran capturar. Es posible también que la población femenina tenga una motivación «más instrumental» y preocupada por los resultados de los exámenes, estudia para ellos pero no se preocupa tanto por la tarea diaria que representan los ejercicios en WebCT, aunque esta hipótesis no se correlaciona con el hecho de que la población femenina interacciona en clase y voluntariamente interacciona con el profesor. El mismo docente valora positivamente la participación de la población femenina en su clase. Otra hipótesis plausible sería que la población femenina cree que el aprendi-

${ }^{2}$ Este examen es de elección múltiple con corrección mediante máquina que da el resultado en tantos por ciento. 
zaje de la lengua se realiza a través de la interacción cara a cara y es allí donde «ellas» desarrollan sus estrategias de aprendizaje que luego se ven reflejadas en los exámenes orales pero no en los ejercicios en WebCT.

Todas estas son algunas de las posibles interpretaciones de los datos que aquí se presentan. Estas conclusiones son una aproximación analítica de los resultados que se han recogido en cada caso. Seguidamente, en la figura I se ilustra la correlación en el caso de la población masculina.

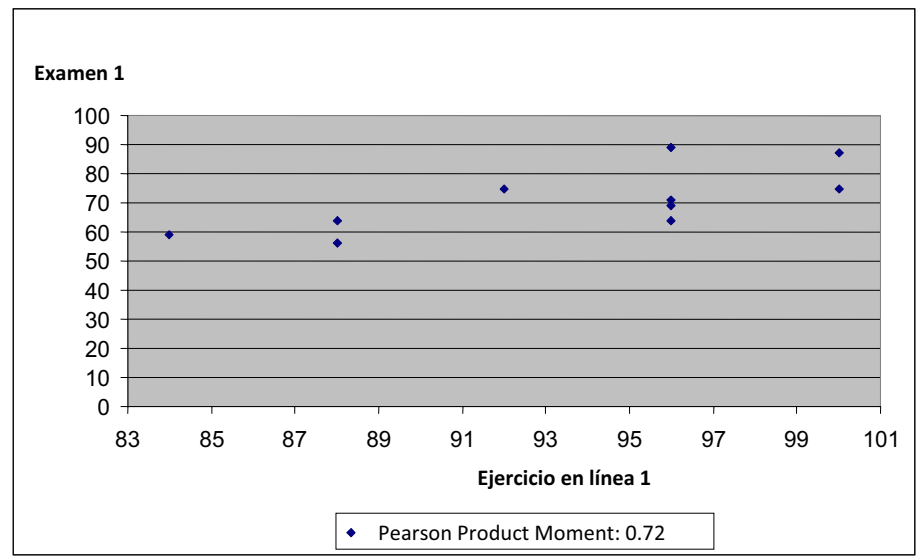

Figura I. Correlación de la población masculina entre los ejercicios en WebCT y el examen 1

Como se muestra en la gráfica, la correlación es positiva y ascendente para los alumnos; pero en el caso de la población femenina (figura II), la correlación es negativa y descendente. Las gráficas en ambos casos son completamente opuestas mostrando una correlación directamente proporcional en un caso, y en otro, una inversamente proporcional.

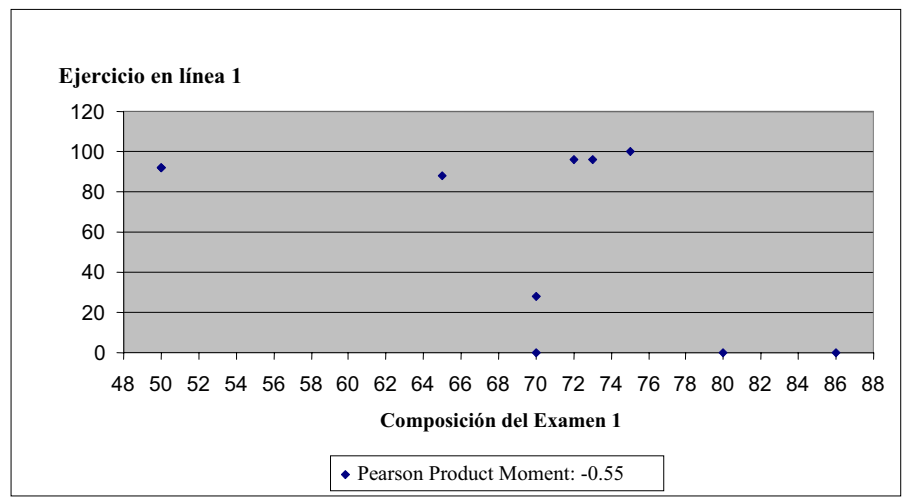

Figura II. Correlación de la población femenina entre los ejercicios en WebCT y la composición del examen 1 


\section{Conclusión}

Si bien hay aún muchos puntos por profundizar en el análisis que se ha presentado aquí; seguidamente se intentará dar respuesta a las preguntas que se formularon al comienzo del estudio.

En relación a la primera pregunta, si los estudiantes de SPAN 2301 que pasan más tiempo practicando el vocabulario en los ejercicios en la plataforma educativa WebCT obtienen mejores resultados que los que practican menos tiempo, se puede responder que sí en el caso de la población masculina, pero esto no se verifica en la población femenina.

En relación a la segunda pregunta, si la cantidad de veces que los estudiantes hacen los ejercicios en línea les ayuda a aprender mejor el vocabulario y si esto se refleja en los exámenes, la respuesta a estas preguntas es afirmativa sólo en el caso de la población masculina. En caso de la población femenina la correlación es baja o negativa.

Con respecto a la tercera pregunta, si existe una correlación positiva entre las notas que los estudiantes obtienen en los ejercicios en la plataforma educativa WebCT, las notas de los exámenes y el tiempo que trabajan en la plataforma educativa WebCT, se puede decir que efectivamente hay una correlación entre todos estos elementos en la población masculina, no así en la población femenina.

Por último, a la pregunta de si existe un «efecto de género» en el rendimiento académico de los estudiantes tanto en la plataforma educativa WebCT como en los exámenes, la respuesta es que podemos concluir que hay un «efecto de género» que se manifiesta en cada uno de los análisis presentados hasta ahora. Los resultados obtenidos en este estudio se corresponden con los de Jiménez Catalán (2003) en tanto ella señala que los alumnos y las alumnas emplean estrategias diferentes para aprender el vocabulario en una L2. Aparentemente, el diseño de los ejercicios en la plataforma educativa WebCT favorecería estrategias masculinas de aprendizaje de vocabulario como L2. Una hipótesis a investigar es que tal vez la población femenina pone en juego durante este proceso estrategias más complejas en la adquisición del vocabulario que la población masculina y éstas no están reflejadas en los ejercicios en la plataforma educativa WebCT. Otra hipótesis a explorar es que las estrategias que la población femenina ponen en juego están ligadas a la interacción cara a cara con el profesor y con sus compañeros y compañeras de clase $\mathrm{y}$, por ese motivo, las alumnas tienen un mejor rendimiento que los alumnos en los exámenes orales, participan más en clase y no superan los ejercicios en la plataforma educativa WebCT tan bien como la población masculina.

Estos resultados no son concluyentes; por el contrario, pretenden prestar atención a las diferencias de género en el aula en el contexto del aprendizaje de segundas lenguas. Es necesario continuar explorando estas problemáticas, en particular el papel que juegan los ejercicios en las plataformas Web y en las distintas plataformas electrónicas que hoy pueblan el mercado editorial. Otros estudios en esta misma línea podrían evaluar el rol de la tecnología y su diseño en el aprendizaje de segundas lenguas y su impacto a la hora de construir patrones de aprendizaje que favorezcan a todos los aprendices por igual. Para que la coeducación sea una realidad en el contexto del aprendizaje de segundas lenguas es necesario continuar investigando los usos de la tecnología y su impacto en el aula. 


\section{Bibliografía}

Chang, R. \& Smith, F. (1991). «Cooperative learning and CALL/IVD in beginning Spanish: An experiment», in The Modern Language Journal 75, 2: 205-211.

Chapelle, C. (1994). «CALL activities: Are they all the same?» System 22, 1: 33-45.

- (1997). "CALL in year 2000: Still in search for research paradigms?», in Language Learning \& Technology 1, 1: 19-43.

- (1998). «Multimedia CALL: Lessons to be learned from research on instructed SLA», in Language Learning \& Technology 2, 1: 22-34.

- (2001). Computer Applications in Second Language Acquisition. Cambridge: Cambridge University Press.

Chun, M. (1994). «Signal analysis software for teaching discourse intonation», in Language Learning \& Technology 2, 1: 61-77.

De la Fuente, M. (2003). «Is SLA integrationist theory relevant to CALL? A study on the effects of computer-mediated interaction in L2 vocabulary acquisition», in Computer Assisted Language Learning, 16, 1: 47-81.

Dodigovic, M. (2005). Artificial Intelligence in Second Language Learning. Raising Error Awareness. New York: Multilingual Matters Ltd.

Dunkel, P. (1991). "The effectiveness of research on computer-assisted instruction and computerassisted language learning», in P. Dunkel (ed.), Computer-Assisted Language Learning and Testing, New York: Newbury House, 5-36.

Jiménez Catalán, R. (2003) «Sex differences in L2 vocabulary learning strategies», in International Journal of Applied Linguistics, 13, 1: 54-77

- (2005). «Linking gender and second language education in a database», in CAUCE, Revista Internacional de Filología y su Didáctica, 28: 205-218.

Kitade, K. (2000). «L2 Learners' discourse and SLA theories in CMC: collaborative interaction in internet chat», in Computer Assisted Language Learning 13, 2: 143-166.

Levy, M. (1997). Computer Assisted Language Learning. Context and Conceptualization. Oxford: Clarendon Press.

Long, M. (1996). «The role of the linguistic environment in second language acquisition», in W. C. Ritchie and T. K. Bathia (eds.), Handbook of Second Language Acquisition. San Diego: Academic Press, 413-68.

Pavlenko, A. (1999). «New approaches to concepts in bilingual memory», Bilingualism: Language and Cognition 2: 209-230.

Pellettieri, J. (2000). «Negotiation in cyberspace: The role of chatting in the development of grammatical competence», in M. Warschaver \& R. Kern (eds.). Network-based Language Teaching: Concepts and Practice. Cambridge: Cambridge University Press, 59-86.

Salaberry, M. (1999). «CALL in year 2000: Still in search for research agenda», in Language Learning \& Technology 1, 3: 104-7.

Singleton, D. (1999). Exploring the second language mental lexicon. Cambridge: Cambridge University Press.

Sun, Y; Dong, Q. (2004). An experiment on supporting children's English vocabulary Learning in Multimedia Context Computer Assisted Language Learning, 17, 2: 131-137.

Swain, M. (2000). «Focus on form through conscious reflection», in C. Doughty and J. Williams (eds.), Focus on form in classroom Second Language Acquisition. New York: Cambridge University Press, pp. 64-82.

Tozcu, A y Coady, J. (2004). «Successful learning of frequent vocabulary through CALL also benefits reading comprehension and speed», in Computer Assisted Languages Learning, 17: 473-495. 